\title{
Early Career Researchers' Perspective on Citizen Science
}

\author{
Petra Biberhofer ${ }^{a} *$, Birte Lembke-Ibold ${ }^{b *}$, Susanne Tönsmann ${ }^{a *}$ \\ a Participatory Science Academy, University of Zurich and ETH Zurich \\ Kurvenstrasse 17, 8006 Zurich, Switzerland \\ $b$ Graduate Campus, University of Zurich, \\ Rämistrasse 59, 8001 Zurich, Switzerland \\ E-mail: petra.biberhofer@fwf.ac.at,birte.lembke@grc.uzh.ch,susanne.toensmann@uzh.ch
}

How do Early Career Researchers (ECR) perceive Citizen Science (CS)? What are specific challenges for researchers at this career stage? By addressing questions on how CS is perceived in the scientific community, this contribution tackles a core topic of the conference call, namely the meaning of CS in the broader academic setting. It discusses findings from a Citizen Science Winter School held in Zurich, Switzerland. Three dimensions are defined as particular challenging for ECR and as in need of concrete measures to support CS in its goal to open up science: competencies and attitudes, academic culture, and institutional opportunities.

Vienna - Austria 


\section{Introduction}

Citizen Science (CS) is a dynamic research field. It implies reinterpreting the role of researchers towards promoting active collaboration and better convergence of actions for science, between researchers, policy makers and the general public [1]. The ambitious standards of CS provide new opportunities to enrich the university curriculum. The goal would be to enhance the ability of researchers, at an early career stage, to learn both how to build CS projects and how to engage the wider public [2], i.e. what we call co-research performance (see Graphic).

At the same time, CS adoption raises many doubts regarding cost, efficiency, openness or length of research. Citizen Science may still be considered a risky endeavour at all stages of an academic career. Especially engaging in CS at early career stages involves uncertainties that may have an impact different to more senior researchers. The aim of this contribution is therefore to better understand the perception of CS in the academic community from an early career researchers $(\mathrm{ECR})$ perspective. In particular, we address the following questions:

- How do ECR perceive CS with regard to planning an academic career in higher education?

- What are obstacles to engaging with CS for ECR in personal, institutional and structural terms?

- What is needed to facilitate ECR engagement with CS; what are their needs and wishes?

The contribution draws on the insights gained from a one-week CS Winter School held at the University of Zurich and ETH Zurich in January 2020 in which 29 international participants (doctoral students, postdocs and practitioners) from 9 different countries took part. Most participants pursue their research in social science and economics $(44 \%)$, others in natural sciences (19\%), engineering (15\%), arts \& humanities (11\%), medicine (7\%) and theology (4\%). In particular, findings are based on a full-day workshop «Early Career Researchers' Perspectives on Citizen Science» which, using LEGO serious play methods, was devoted to discussing challenges of interest to doctoral students and early postdocs. In addition, evaluation results from reflection sessions and a survey conducted with all participants of the Winter School were consulted.

\section{Findings}

Participants highlight challenges for ECR engaged in CS and respective measures on three dimensions: (1) key competencies for CS and corresponding attitudes, (2) the dominant academic culture as a set of values and motivations, and (3) opportunities in terms of institutional/structural framework conditions. The graphic shows the interrelations of these three dimensions influencing the perspective of ECR to engage in CS. This is referred to as co-research performance. Coresearch performance depends on competencies, understood as specific attributes individuals need for action and self-organization. Competencies include cognitive, affective, volitional and motivational elements and are an interplay of knowledge, capacities and skills, motives and affective dispositions [3]. Besides knowledge and skills, values and motivation are crucial when it comes to fostering key competencies for CS. Furthermore, the environment in which ECR work was emphasised as challenging because opportunities i.e. environmental and contextual 
mechanisms to perform are beyond the individual's control [4]. The academic culture and institutional conditions were identified as the most important potentially encouraging or discouraging opportunity factors that enable action. In the following, we discuss these dimensions in more detail.

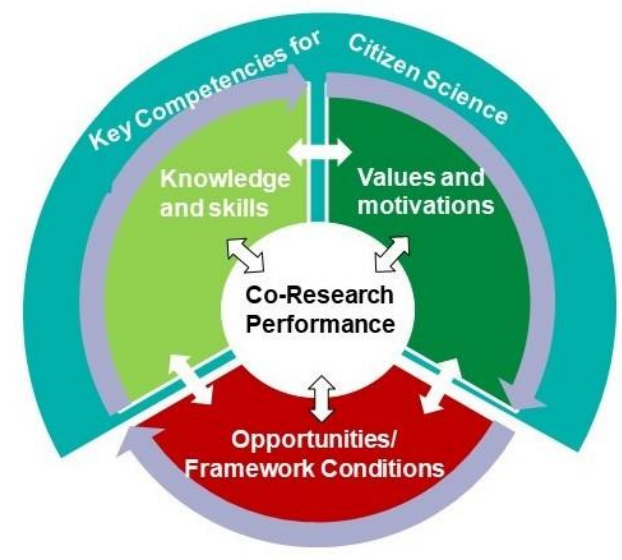

Graphic: Key competencies and performance of ECR (based on [4] adapted by the authors)

\subsection{Key competencies for CS and corresponding attitudes}

From an ECR perspective CS requires a broad set of competencies, including collaborative competencies (being able to interact in heterogeneous groups, communicate and translate between different mindsets) and normative competencies (implicit assumptions made explicit). In addition, anticipatory competencies, including the ability to cope with uncertainties. An observation shared during the winter school is that doctoral students who engage in CS as part of their doctoral program graduate with a broader set of competencies and skills than peers [5]. ECR view these key competencies as linked to a mindset that includes the ability to be aware of one's feelings, and those of a group as having an impact on a research process.

We argue that these attributes may not be consciously recognized and often remain unexamined in daily CS practice, but that they are influential and impactful. For competenceoriented curriculum development it seems important to recognize that beyond knowledge and skills about CS, individuals need corresponding values and motivational drivers to engage in CS.

\subsection{CS and the dominant academic culture}

Young researchers report a lack of appreciation of CS in the academic culture. They pointed to the perception of CS as potentially "less scientific" in the academic community. This includes doubts about data quality or the lack of trust in the abilities of citizen scientists. In addition, the dominance of "academic dinosaurs" i.e. a persistent habitus was highlighted as an obstructionist factor to enact the countercultural potential of CS. For instance, academic hierarchies are not conducive to an appreciation of expertise that is produced outside of academia, or that stems from experience rather than method and theory-driven research.

ECR also mention that there are few role models in their own discipline or their own university who undertake CS projects and associated activities. Outreach activities and other communicative efforts are commonly not considered a relevant skill and part of the recognised academic performance, instead they are perceived as "nice to have". 
Fundamental shifts in the academic culture are seen as necessary. Taking into account that such shifts require complex and long-term processes, possible measures and steps towards more appreciation of these elements were discussed and are spelled out below.

\subsection{Structural/institutional conditions for ECR and CS}

Doctorates have a narrow time frame, with little room for time delays. As the length of phases for CS projects is often different compared to traditional research projects, ECR perceive the narrow time frame as rather challenging. In addition, few universities offer creditable courses (e.g. on methodology or process support) and training opportunities.

While the support of a supervisor can provide critical benefit, the absence of a committed supervisor makes a supportive institutional environment and academic culture all the more important.

Universities usually do not provide decision-making competence for citizens. However, the relevance of learning in and from diverse groups (academics, practitioners, people, citizens) was mentioned to be crucial for ECR co-research performance (see above collaboration competencies as a key competence for CS).

As a rule, citizens are not entitled to apply for funding from national research funding agencies. Universities have limited advisory competence for CS projects (funding, communication).

In sum, bringing about change in institutional practices and academic culture was discussed as a multi-faceted, long-term perspective.

\section{Concrete measures and steps}

There were concrete measures identified that would motivate ECR to engage in CS:

Recognition: Acknowledging success through supervisors or the university as an institution by way of awarding prizes or awards for CS projects.

Funding: Funding schemes for CS or a CS component were seen as conducive to the possibility to engage in $\mathrm{CS}$ and to increase its prestige.

Impact orientation: Participants discussed CS in terms of the impact of science, suggesting that a definition of impact that includes societal and social impact was favorable to a broader acceptance of CS.

Training: The integration of inter- and transdisciplinary CS-Trainings - by way of examples of projects or training of skills important for CS at all career levels was highlighted as a way to train a new generation of scientists for whom CS becomes a valid approach to science.

\section{References}

[1] Hecker S., Haklay, M., Bowser, A., Makuch, Z., Vogel, J., and Bonn, A. (Eds.) (2018) Citizen Science. Innovation in Open Science, Society and Policy. UCL Press. Download Free. 
[2] Bonn A. et al., Citizen science to foster innovation in open science, society and policy. In Hecker, S., Haklay, M., Bowser, A., Makuch, Z., Vogel, J., and Bonn, A. (Eds.) (2018) Citizen Science. Innovation in Open Science, Society and Policy. UCL Press. Download Free. (465-484)

[3] UNESCO (2017) Education for Sustainable Development Goals Learning Objectives. Report, United Nations Educational, Scientific and Cultural Organization, Paris. http://unesdoc.unesco.org/images/0024/002474/247444e.pdf

[4] Rieckmann, M. Chapter 2: Learning to transform the world: key competencies in Education for Sustainable Development. In Leicht, A., Heiss, J. and Byun, W. J. (eds) (2018) Issues and trends in Education for Sustainable Development. Unesco Publishing.

[5] Seibert, Jan (2020) Remarks during the Workshop "Supporting Citizen Science in Academy and beyond" at ETH Zurich, January 16, 2020. 